\title{
ON THE RECIPROCAL SUMS OF SQUARE OF GENERALIZED BI-PERIODIC FIBONACCI NUMBERS
}

\author{
GINKYU CHOI AND YOUNSEOK CHOO
}

Received 06 September, 2017

\begin{abstract}
Recently Basbük and Yazlik [1] proved identities related to the reciprocal sum of generalized bi-periodic Fibonacci numbers starting from 0 and 1, and raised an open question whether we can obtain similar results for the reciprocal sum of $m^{\text {th }}$ power $(m \geq 2)$ of the same numbers. In this paper we derive identities for the reciprocal sum of square of generalized biperiodic Fibonacci numbers with arbitrary initial conditions.
\end{abstract}

2010 Mathematics Subject Classification: 11B39; 11B37

Keywords: bi-periodic Fibonacci numbers, reciprocal sum, floor function

\section{INTRODUCTION}

Throughout this paper we use the notation $\left\{G_{n}\right\}_{n=0}^{\infty}=S\left(G_{0}, G_{1}, a, b\right)$ to denote the generalized bi-periodic Fibonacci numbers $\left\{G_{n}\right\}_{n=0}^{\infty}$ generated from the recurrence relation [4]

$$
G_{n}=\left\{\begin{array}{ll}
a G_{n-1}+G_{n-2}, & \text { if } n \in \mathbb{N}_{e} ; \\
b G_{n-1}+G_{n-2}, & \text { if } n \in \mathbb{N}_{o},
\end{array} \quad(n \geq 2),\right.
$$

with initial conditions $G_{0}$ and $G_{1}$, where $G_{0}, G_{1}, a$ and $b$ are real numbers, and $\mathbb{N}_{e}$ $\left(\mathbb{N}_{o}\right.$, respectively) denotes the set of positive even (odd, respectively) integers.

Recently Ohtsuka and Nakamura [8] found interesting properties of the Fibonacci numbers $\left\{F_{n}\right\}_{n=0}^{\infty}=S(0,1,1,1)$ and proved (1.1) and (1.2) below, where $\lfloor\cdot\rfloor$ indicates the floor function.

$$
\begin{gathered}
\left\lfloor\left(\sum_{k=n}^{\infty} \frac{1}{F_{k}}\right)^{-1}\right\rfloor=\left\{\begin{array}{l}
F_{n}-F_{n-1}, \text { if } n \geq 2 \text { and } n \in \mathbb{N}_{e} ; \\
F_{n}-F_{n-1}-1, \text { if } n \geq 3 \text { and } n \in \mathbb{N}_{o},
\end{array}\right. \\
\left\lfloor\left(\sum_{k=n}^{\infty} \frac{1}{F_{k}^{2}}\right)^{-1}\right\rfloor=\left\{\begin{array}{l}
F_{n-1} F_{n}-1, \text { if } n \geq 2 \text { and } n \in \mathbb{N}_{e} ; \\
F_{n-1} F_{n}, \text { if } n \geq 3 \text { and } n \in \mathbb{N}_{o} .
\end{array}\right.
\end{gathered}
$$


The work of Ohtsuka and Nakamura was generalized by several authors [1,2,5-7]. In particular, Basbük and Yazlik [1] considered the reciprocal sum of generalized biperiodic Fibonacci numbers $\left\{G_{n}\right\}_{n=0}^{\infty}=S(0,1, a, b)$ and proved the following theorem.

Theorem 1. Let $a$ and $b$ positive integers. Then, for $\left\{G_{n}\right\}_{n=0}^{\infty}=S(0,1, a, b)$, we have

$$
\left\lfloor\left(\sum_{k=n}^{\infty} \frac{\left(\frac{a}{b}\right)^{\psi(k)}}{G_{k}}\right)^{-1}\right\rfloor=\left\{\begin{array}{l}
G_{n}-G_{n-1}, \text { if } n \geq 2 \text { and } n \in \mathbb{N}_{e} \\
G_{n}-G_{n-1}-1, \text { if } n \geq 1 \text { and } n \in \mathbb{N}_{o}
\end{array}\right.
$$

where

$$
\psi(k)=\xi(k+1)-\xi(n+1)-(-1)^{n}\left\lfloor\frac{k-n}{2}\right\rfloor,
$$

and $\xi(n)$ is the parity function such that

$$
\xi(n)=\left\{\begin{array}{l}
0, \text { if } n \in\{0\} \cup \mathbb{N}_{e} \\
1, \text { if } n \in \mathbb{N}_{o}
\end{array}\right.
$$

In [1], Basbük and Yazlik raised an open question whether we can obtain similar results for the reciprocal sum of $m^{\text {th }}$ power $(m \geq 2)$ of the same numbers.

In this paper we derive identities for the reciprocal sum of square of generalized biperiodic Fibonacci numbers $\left\{G_{n}\right\}_{n=0}^{\infty}=S\left(G_{0}, G_{1}, a, b\right)$, where $G_{0}$ is a nonnegative integer and $G_{1}$ is a positive integer.

\section{MAIN RESULTS}

Lemma 1 below will be used to prove our main results.

Lemma 1. For $\left\{G_{n}\right\}_{n=0}^{\infty}=S\left(G_{0}, G_{1}, a, b\right),(a)$-(c) below hold:

(a) $G_{n} G_{n+1}-G_{n-1} G_{n+2}=(-1)^{n}\left(b G_{0}^{2}+a b G_{0} G_{1}-a G_{1}^{2}\right)$.

(b) $a^{\xi(n+1)} b^{\xi(n)} G_{n-1} G_{n+1}-a^{\xi(n)} b^{\xi(n+1)} G_{n}^{2}=(-1)^{n}\left(a G_{1}^{2}-a b G_{0} G_{1}-b G_{0}^{2}\right)$.

(c) $G_{n+1} G_{n+2}-G_{n-1} G_{n}=a^{\xi(n)} b^{\xi(n+1)} G_{n}^{2}+a^{\xi(n+1)} b^{\xi(n)} G_{n+1}^{2}$.

Proof. (a) and (b) are special cases of [3, Theorem 2.2]. Since

$$
G_{n}=a^{\xi(n-1)} b^{\xi(n)} G_{n-1}+G_{n-2},
$$

then (c) follows from the identity

$$
\begin{aligned}
G_{n} G_{n+1} & =\left(G_{n+2}-a^{\xi(n+1)} b^{\xi(n)} G_{n+1}\right) G_{n+1} \\
& =G_{n}\left(a^{\xi(n)} b^{\xi(n+1)} G_{n}+G_{n-1}\right) .
\end{aligned}
$$


The main results of this paper are stated in Theorem 2. For the ease of presentation, we use the following notation for $\left\{G_{n}\right\}_{n=0}^{\infty}=S\left(G_{0}, G_{1}, a, b\right)$

$$
\Phi(G):=b^{2} G_{0}^{2}+a b^{2} G_{0} G_{1}-a b G_{1}^{2} .
$$

Theorem 2. Let $G_{0}$ be a nonnegative integer and let $G_{1}$, $a$ and $b$ positive integers. Then, for $\left\{G_{n}\right\}_{n=0}^{\infty}=S\left(G_{0}, G_{1}, a, b\right)$, (a) and (b) below hold:

(a) If

$$
\frac{\Phi(G)}{a b+2} \notin \mathbb{Z}
$$

define

$$
g:=\left\lfloor\frac{\Phi(G)}{a b+2}\right\rfloor+\Delta
$$

where

$$
\Delta=\left\{\begin{array}{l}
1, \text { if } \Phi(G)>0 \\
0, \text { if } \Phi(G)<0
\end{array}\right.
$$

(i) If $\Phi(G)>0$, then there exist positive integers $n_{0}$ and $n_{1}$ such that

$$
\left\lfloor\left(\sum_{k=n}^{\infty} \frac{\left(\frac{a}{b}\right)^{1-\xi(k)}}{G_{k}^{2}}\right)^{-1}\right\rfloor=\left\{\begin{array}{l}
b G_{n-1} G_{n}+g-1, \text { if } n \geq n_{0} \text { and } n \in \mathbb{N}_{e} \\
b G_{n-1} G_{n}-g, \text { if } n \geq n_{1} \text { and } n \in \mathbb{N}_{o} .
\end{array}\right.
$$

(ii) If $\Phi(G)<0$, then there exist positive integers $n_{2}$ and $n_{3}$ such that

$$
\left\lfloor\left(\sum_{k=n}^{\infty} \frac{\left(\frac{a}{b}\right)^{1-\xi(k)}}{G_{k}^{2}}\right)^{-1}\right\rfloor=\left\{\begin{array}{l}
b G_{n-1} G_{n}+g, \text { if } n \geq n_{2} \text { and } n \in \mathbb{N}_{e} \\
b G_{n-1} G_{n}-g-1, \text { if } n \geq n_{3} \text { and } n \in \mathbb{N}_{o}
\end{array}\right.
$$

(b) If

$$
\frac{\Phi(G)}{a b+2} \in \mathbb{Z}
$$

then there exist positive integers $n_{4}$ and $n_{5}$ such that

$$
\left\lfloor\left(\sum_{k=n}^{\infty} \frac{\left(\frac{a}{b}\right)^{1-\xi(k)}}{G_{k}^{2}}\right)^{-1}\right\rfloor=\left\{\begin{array}{l}
b G_{n-1} G_{n}+\hat{g}, \text { if } n \geq n_{4} \text { and } n \in \mathbb{N}_{e} \\
b G_{n-1} G_{n}-\hat{g}, \text { if } n \geq n_{5} \text { and } n \in \mathbb{N}_{o}
\end{array}\right.
$$

where

$$
\hat{g}:=\frac{\Phi(G)}{a b+2} .
$$

Proof. (a) To prove (2.1), assume that $\Phi(G)>0$. Then

$$
\Phi(G)-g(a b+2)<0 .
$$

Firstly, consider 


$$
\begin{gathered}
X_{1}=\frac{1}{b G_{n-1} G_{n}+(-1)^{n} g}-\frac{1}{b G_{n+1} G_{n+2}+(-1)^{n} g} \\
-\frac{\left(\frac{a}{b}\right)^{1-\xi(n)}}{G_{n}^{2}}-\frac{\left(\frac{a}{b}\right)^{1-\xi(n+1)}}{G_{n+1}^{2}} \\
=\frac{Y_{1}}{\left(b G_{n-1} G_{n}+(-1)^{n} g\right)\left(b G_{n+1} G_{n+2}+(-1)^{n} g\right) G_{n}^{2} G_{n+1}^{2}},
\end{gathered}
$$

where, by Lemma $1(\mathrm{c})$

$$
Y_{1}=\left\{\left(\frac{a}{b}\right)^{1-\xi(n+1)} G_{n}^{2}+\left(\frac{a}{b}\right)^{1-\xi(n)} G_{n+1}^{2}\right\} \hat{Y}_{1},
$$

with

$$
\begin{array}{r}
\hat{Y}_{1}=b^{2}\left(G_{n}^{2} G_{n+1}^{2}-G_{n-1} G_{n} G_{n+1} G_{n+2}\right) \\
\quad-(-1)^{n} g b\left(G_{n-1} G_{n}+G_{n+1} G_{n+2}\right)-g^{2} .
\end{array}
$$

By Lemma 2.1(a),(b), we have

$$
\begin{aligned}
G_{n}^{2} G_{n+1}^{2}-G_{n-1} G_{n} G_{n+1} G_{n+2} & \\
=\left(G_{n} G_{n+1}-\right. & \left.G_{n-1} G_{n+2}\right) G_{n} G_{n+1} \\
& =(-1)^{n}\left(b G_{0}^{2}+a b G_{0} G_{1}-a G_{1}^{2}\right) G_{n} G_{n+1},
\end{aligned}
$$

and

$$
\begin{aligned}
& G_{n-1} G_{n}+G_{n+1} G_{n+2} \\
& =\left(G_{n+1}-a^{\xi(n)} b^{\xi(n+1)} G_{n}\right) G_{n}+G_{n+1}\left(a^{\xi(n+1)} b^{\xi(n)} G_{n+1}+G_{n}\right) \\
& =(a b+2) G_{n} G_{n+1}+a^{\xi(n+1)} b^{\xi(n)} G_{n-1} G_{n+1}-a^{\xi(n)} b^{\xi(n+1)} G_{n}^{2} \\
& \quad=(a b+2) G_{n} G_{n+1}+(-1)^{n}\left(a G_{1}^{2}-a b G_{0} G_{1}-b G_{0}^{2}\right) .
\end{aligned}
$$

Then

$$
\begin{aligned}
\hat{Y}_{1}= & (-1)^{n} b^{2}\left(b G_{0}^{2}+a b G_{0} G_{1}-a G_{1}^{2}\right) G_{n} G_{n+1} \\
& -(-1)^{n} g b\left\{(a b+2) G_{n} G_{n+1}+(-1)^{n}\left(a G_{1}^{2}-a b G_{0} G_{1}-b G_{0}^{2}\right)\right\}-g^{2} \\
& =(-1)^{n} b G_{n} G_{n+1}\{\Phi(G)-g(a b+2)\}+g \Phi(G)-g^{2} .
\end{aligned}
$$

If $n \in \mathbb{N}_{e}$, then there exists a positive integer $m_{0}$ such that, for $n \geq m_{0}, X_{1}<0$, and

$$
\frac{1}{b G_{n-1} G_{n}+(-1)^{n} g}-\frac{1}{b G_{n+1} G_{n+2}+(-1)^{n} g}<\frac{\left(\frac{a}{b}\right)^{1-\xi(n)}}{G_{n}^{2}}+\frac{\left(\frac{a}{b}\right)^{1-\xi(n+1)}}{G_{n+1}^{2}} .
$$


Repeatedly applying the above inequality, we have

$$
\frac{1}{b G_{n-1} G_{n}+(-1)^{n} g}<\sum_{k=n}^{\infty} \frac{\left(\frac{a}{b}\right)^{1-\xi(k)}}{G_{k}^{2}} \text {, if } n \geq m_{0} \text { and } n \in \mathbb{N}_{e} .
$$

Similarly, we obtain, for some positive integer $m_{1}$,

$$
\sum_{k=n}^{\infty} \frac{\left(\frac{a}{b}\right)^{1-\xi(k)}}{G_{k}^{2}}<\frac{1}{b G_{n-1} G_{n}+(-1)^{n} g} \text {, if } n \geq m_{1} \text { and } n \in \mathbb{N}_{o} .
$$

Next, consider

$$
\begin{aligned}
& X_{2}=\frac{1}{b G_{n-1} G_{n}+(-1)^{n} g-1}-\frac{1}{b G_{n} G_{n+1}+(-1)^{n+1} g-1}-\frac{\left(\frac{a}{b}\right)^{1-\xi(n)}}{G_{n}^{2}} \\
& =\frac{Y_{2}}{\left(b G_{n-1} G_{n}+(-1)^{n} g-1\right)\left(b G_{n} G_{n+1}+(-1)^{n+1} g-1\right) G_{n}^{2}},
\end{aligned}
$$

where

$$
\begin{aligned}
Y_{2}= & b G_{n}^{3} G_{n+1}-b a^{1-\xi(n)} b^{\xi(n)} G_{n-1} G_{n}^{2} G_{n+1}-b G_{n-1} G_{n}^{3} \\
& -(-1)^{n} g\left(2 G_{n}^{2}-a^{1-\xi(n)} b^{\xi(n)} G_{n-1} G_{n}+a^{1-\xi(n)} b^{\xi(n)} G_{n} G_{n+1}\right) \\
& +a^{1-\xi(n)} b^{\xi(n)}\left(G_{n-1} G_{n}+G_{n} G_{n+1}\right)+a^{1-\xi(n)} b^{\xi(n)-1}\left(g^{2}-1\right) .
\end{aligned}
$$

Using Lemma 1(a), we have

$$
\begin{aligned}
& b G_{n}^{3} G_{n+1}-b a^{1-\xi(n)} b^{\xi(n)} G_{n-1} G_{n}^{2} G_{n+1}-b G_{n-1} G_{n}^{3} \\
& =b G_{n}^{2} G_{n+1}\left(G_{n}-a^{1-\xi(n)} b^{\xi(n)} G_{n-1}\right)-b G_{n-1} G_{n}^{3} \\
& =b G_{n}^{2}\left(G_{n-2} G_{n+1}-G_{n-1} G_{n}\right) \\
& =(-1)^{n} b G_{n}^{2}\left(b G_{0}^{2}+a b G_{0} G_{1}-a G_{1}^{2}\right),
\end{aligned}
$$

and

$$
\begin{aligned}
& 2 G_{n}^{2}-a^{1-\xi(n)} b^{\xi(n)} G_{n-1} G_{n}+a^{1-\xi(n)} b^{\xi(n)} G_{n} G_{n+1} \\
= & 2 G_{n}^{2}-a^{1-\xi(n)} b^{\xi(n)} G_{n-1} G_{n}+a^{1-\xi(n)} b^{\xi(n)} G_{n}\left(a^{1-\xi(n+1)} b^{\xi(n+1)} G_{n}+G_{n-1}\right) \\
& =(a b+2) G_{n}^{2} .
\end{aligned}
$$

Hence we obtain

$$
\begin{aligned}
Y_{2}=(-1)^{n} G_{n}^{2} & \{\Phi(G)-g(a b+2)\} \\
& +a^{1-\xi(n)} b^{\xi(n)}\left(G_{n-1} G_{n}+G_{n} G_{n+1}\right)+a^{1-\xi(n)} b^{\xi(n)-1}\left(g^{2}-1\right) .
\end{aligned}
$$


If $n \in \mathbb{N}_{e}$, then there exists a positive integer $m_{2}$ such that, for $n \geq m_{2}, X_{2}>0$, and

$$
\frac{\left(\frac{a}{b}\right)^{1-\xi(n)}}{G_{n}^{2}}<\frac{1}{b G_{n-1} G_{n}+(-1)^{n} g-1}-\frac{1}{b G_{n} G_{n+1}+(-1)^{n+1} g-1} .
$$

Repeatedly applying the above inequality, we have

$$
\sum_{k=n}^{\infty} \frac{\left(\frac{a}{b}\right)^{1-\xi(k)}}{G_{k}^{2}}<\frac{1}{b G_{n-1} G_{n}+(-1)^{n} g-1} \text {, if } n \geq m_{2} \text { and } n \in \mathbb{N}_{e} .
$$

Similarly, consider

$$
\begin{aligned}
& X_{3}=\frac{1}{b G_{n-1} G_{n}+(-1)^{n} g+1}-\frac{1}{b G_{n} G_{n+1}+(-1)^{n+1} g+1}-\frac{\left(\frac{a}{b}\right)^{1-\xi(n)}}{G_{n}^{2}} \\
& =\frac{Y_{3}}{\left(b G_{n-1} G_{n}+(-1)^{n} g+1\right)\left(b G_{n} G_{n+1}+(-1)^{n+1} g+1\right) G_{n}^{2}},
\end{aligned}
$$

where

$$
\begin{gathered}
Y_{3}=Y_{2}-2 a^{1-\xi(n)} b^{\xi(n)}\left(G_{n-1} G_{n}+G_{n} G_{n+1}\right) \\
=(-1)^{n} G_{n}^{2}\{\Phi(G)-g(a b+2)\} \\
-a^{1-\xi(n)} b^{\xi(n)}\left(G_{n-1} G_{n}+G_{n} G_{n+1}\right)+a^{1-\xi(n)} b^{\xi(n)-1}\left(g^{2}-1\right) .
\end{gathered}
$$

If $n \in \mathbb{N}_{o}$, then there exists a positive integer $m_{3}$ such that, for $n \geq m_{3}, X_{3}<0$, and

$$
\frac{1}{b G_{n-1} G_{n}+(-1)^{n} g+1}-\frac{1}{b G_{n} G_{n+1}+(-1)^{n+1} g+1}<\frac{\left(\frac{a}{b}\right)^{1-\xi(n)}}{G_{n}^{2}},
$$

from which we have

$$
\frac{1}{b G_{n-1} G_{n}+(-1)^{n} g+1}<\sum_{k=n}^{\infty} \frac{\left(\frac{a}{b}\right)^{1-\xi(k)}}{G_{k}^{2}} \text {, if } n \geq m_{3} \text { and } n \in \mathbb{N}_{o} .
$$

Then (2.1) follows from (2.4), (2.5), (2.6) and (2.7).

Now suppose that $\Phi(G)<0$. In this case, we have

$$
\Phi(G)-g(a b+2)>0,
$$

and (2.4), (2.5), (2.6) and (2.7) are respectively modified as

$$
\sum_{k=n}^{\infty} \frac{\left(\frac{a}{b}\right)^{1-\xi(k)}}{G_{k}^{2}}<\frac{1}{b G_{n-1} G_{n}+(-1)^{n} g}, \text { if } n \geq m_{4} \text { and } n \in \mathbb{N}_{e}
$$




$$
\frac{1}{b G_{n-1} G_{n}+(-1)^{n} g}<\sum_{k=n}^{\infty} \frac{\left(\frac{a}{b}\right)^{1-\xi(k)}}{G_{k}^{2}} \text {, if } n \geq m_{5} \text { and } n \in \mathbb{N}_{o},
$$

$$
\sum_{k=n}^{\infty} \frac{\left(\frac{a}{b}\right)^{1-\xi(k)}}{G_{k}^{2}}<\frac{1}{b G_{n-1} G_{n}+(-1)^{n} g-1}, \text { if } n \geq m_{6} \text { and } n \in \mathbb{N}_{o},
$$

and

$$
\frac{1}{b G_{n-1} G_{n}+(-1)^{n} g+1}<\sum_{k=n}^{\infty} \frac{\left(\frac{a}{b}\right)^{1-\xi(k)}}{G_{k}^{2}} \text {, if } n \geq m_{7} \text { and } n \in \mathbb{N}_{e} .
$$

Then, (2.2) easily follows and the proof of (a) is completed.

(b) Suppose that

$$
\frac{\Phi(G)}{a b+2} \in \mathbb{Z}
$$

We recall the proof of (a). Replacing $g$ by $\hat{g}$, we have

$$
\hat{Y}_{1}=\hat{g} \Phi(G)-\hat{g}^{2}=(a b+1) \hat{g}^{2}>0 .
$$

Hence there exist positive integers $m_{8}$ and $m_{9}$ such that $X_{1}>0$ if $n \geq m_{8}$ and $n \in \mathbb{N}_{e}$ or if $n \geq m_{9}$ and $n \in \mathbb{N}_{o}$. Hence we obtain

$$
\sum_{k=n}^{\infty} \frac{\left(\frac{a}{b}\right)^{1-\xi(k)}}{G_{k}^{2}}<\frac{1}{b G_{n-1} G_{n}+(-1)^{n} \hat{g}} \text {, if } n \geq m_{8}\left(n \in \mathbb{N}_{e}\right) \text { or if } n \geq m_{9}\left(n \in \mathbb{N}_{o}\right) \text {. }
$$

Similarly, there exist positive integers $m_{10}$ and $m_{11}$ such that $X_{3}<0$ if $n \geq m_{10}$ and $n \in \mathbb{N}_{e}$ or if $n \geq m_{11}$ and $n \in \mathbb{N}_{o}$, from which we have

$$
\frac{1}{b G_{n-1} G_{n}+(-1)^{n} \hat{g}+1}<\sum_{k=n}^{\infty} \frac{\left(\frac{a}{b}\right)^{1-\xi(k)}}{G_{k}^{2}} \text {, if } n \geq m_{10}\left(n \in \mathbb{N}_{e}\right) \text { or if } n \geq m_{11}\left(n \in \mathbb{N}_{o}\right) \text {. }
$$

Then, (2.3) follows from (2.12) and (2.13), and (b) is also proved.

Example 1. For $\left\{G_{n}\right\}_{n=0}^{\infty}=S(2,1,2,1)$, we have $\Phi(G)=6$ and

$$
g=\left\lfloor\frac{6}{4}\right\rfloor+1=2 \text {. }
$$

Then, from (2.1), we have

$$
\left\lfloor\left(\sum_{k=n}^{\infty} \frac{2^{1-\xi(k)}}{G_{k}^{2}}\right)^{-1}\right\rfloor=\left\{\begin{array}{l}
G_{n-1} G_{n}+1, \text { if } n \geq 2 \text { and } n \in \mathbb{N}_{e} \\
G_{n-1} G_{n}-2, \text { if } n \geq 1 \text { and } n \in \mathbb{N}_{o}
\end{array}\right.
$$


Example 2. Consider $\left\{G_{n}\right\}_{n=0}^{\infty}=S(0,1, a, b)$ with $a$ and $b$ positive integers. In this case, we have $\Phi(G)=-a b<0$ and

$$
g=\left\lfloor\frac{-a b}{a b+2}\right\rfloor=-1
$$

Then, from (2.2), we obtain

$$
\left\lfloor\left(\sum_{k=n}^{\infty} \frac{\left(\frac{a}{b}\right)^{1-\xi(k)}}{G_{k}^{2}}\right)^{-1}\right\rfloor=\left\{\begin{array}{l}
b G_{n-1} G_{n}-1, \text { if } n \geq 2 \text { and } n \in \mathbb{N}_{e} \\
b G_{n-1} G_{n}, \text { if } n \geq 1 \text { and } n \in \mathbb{N}_{o}
\end{array}\right.
$$

Example 3. For $\left\{G_{n}\right\}_{n=0}^{\infty}=S(2,1,4,2)$, we have $\Phi(G)=40, \hat{g}=4$. Then, from (2.3), we have

$$
\left\lfloor\left(\sum_{k=n}^{\infty} \frac{2^{1-\xi(k)}}{G_{k}^{2}}\right)^{-1}\right\rfloor=\left\{\begin{array}{l}
2 G_{n-1} G_{n}+4, \text { if } n \geq 2 \text { and } n \in \mathbb{N}_{e} \\
2 G_{n-1} G_{n}-4, \text { if } n \geq 1 \text { and } n \in \mathbb{N}_{o}
\end{array}\right.
$$

\section{ACKNOWLEDGEMENT}

The authors thank to the anonymous reviewer for his helpful comments which led to improved presentation of the paper.

\section{REFERENCES}

[1] M. Basbük and Y. Yazlik, "On the sum of reciprocal of generalized bi-periodic Fibonacci numbers." Miskolc Math. Notes., vol. 17, no. 1, pp. 35-41, 2016, doi: 10.18514/MMN.2016.1667.

[2] Y. Choo, "On the reciprocal sums of generalized Fibonacci numbers." Int. J. Math. Anal., vol. 10, no. 28, pp. 1365-1373, 2016, doi: https://doi.org/ 10.12988/ijma.2016.610118.

[3] Y. Choo, "On the generalizations of Fibonacci identities." Results. Math., vol. 71, no. 1-2, pp. 347-356, 2017, doi: 10.1007/s00025-015-0515-6.

[4] E. Edson and O. Yayenie, "A new generalization of Fibonacci sequences and extended Binet's formula." Integers, vol. 9, pp. 639-654, 2009, doi: 10.1515/INTEG.2009.051.

[5] S. Holliday and T. Komatsu, "On the sum of reciprocal generalized Fibonacci numbers." Integers, vol. 11A, p. Article 11, 2011, doi: 10.1515/INTEG.2009.051.

[6] E. Kilic and T. Arican, "More on the infinite sum of reciprocal Fibonacci, Pell and higher order recurrences." Appl. Math. Comput., vol. 219, pp. 7783-7788, 2013, doi: http://doi.org/10.1016/j.amc.2013.02.003.

[7] T. Komatsu and V. Laohakosol, "On the sum of reciprocals of numbers satisfying a recurrence relation of order s." J. Integer Seq., vol. 13, no. 5, p. Article 10.5.8, 2010, doi: http://doi.org/10.1016/j.amc.2013.02.003.

[8] H. Ohtsuka and S. Nakamura, "On the sum of reciprocal Fibonacci numbers." Fibonacci Quart., vol. 46/47, no. 2, pp. 153-159, 2008/2009, doi: http://doi.org/10.1016/j.amc.2013.02.003. 
Authors' addresses

\section{Ginkyu Choi}

Hongik University, Department of Electronic and Electrical Engineering, 2639 Sejong-Ro, 30016 Sejong, Republic of Korea

E-mail address: gkchoi@hongik.ac.kr

Younseok Choo

Hongik University, Department of Electronic and Electrical Engineering, 2639 Sejong-Ro, 30016 Sejong, Republic of Korea

E-mail address: yschoo@hongik.ac.kr 